\title{
E2F1 induces TINCR transcriptional activity and accelerates gastric cancer progression via activation of TINCR/STAU1/CDKN2B signaling axis
}

\author{
Tong-Peng $\mathrm{Xu}^{1,8}$, Yan-Fen Wang ${ }^{2,8}$, Wei-Liang Xiong ${ }^{3}$, Pei Ma ${ }^{1}$, Wen-Yu Wang ${ }^{1}$, Wen-Ming Chen ${ }^{4}$, Ming-De Huang ${ }^{5}$, Rui Xia ${ }^{6}$,
} Rong Wang ${ }^{1}$, Er-Bao Zhang ${ }^{7}$, Yan-Wen Liu ${ }^{7}$, Wei De ${ }^{7}$ and Yong-Qian Shu ${ }^{*, 1}$

Recent evidence indicates that E2F1 transcription factor have pivotal roles in the regulation of cellular processes, and is found to be dysregulated in a variety of cancers. Long non-coding RNAs (IncRNAs) are also reported to exert important effect on tumorigenesis. E2F1 is aberrantly expressed in gastric cancer (GC), and biology functions of E2F1 in GC are controversial. The biological characteristics of E2F1 and correlation between E2F1 and IncRNAs in GC remain to be found. In this study, integrated analysis revealed that E2F1 expression was significantly increased in GC cases and its expression was positively correlated with the poor pathologic stage, large tumor size and poor prognosis. Forced E2F1 expression promotes proliferation, whereas loss of E2F1 function decreased cell proliferation by blocking of cell cycle in GC cells. Mechanistic analyses indicated that E2F1 accelerates GC growth partly through induces TINCR transcription. TINCR could bind to STAU1 (staufen1) protein, and influence CDKN2B mRNA stability and expression, thereby affecting the proliferation of GC cells. Together, our findings suggest that E2F1/TINCR/STAU1/CDKN2B signaling axis contributes to the oncogenic potential of GC and may constitute a potential therapeutic target in this disease.

Cell Death and Disease (2017) 8, e2837; doi:10.1038/cddis.2017.205; published online 1 June 2017

Gastric cancer (GC) is still one of the most significant health problems in the world with particularly high frequencies in East Asia. ${ }^{1}$ The roles of genetic dysregulation, epigenetic changes and signaling pathways involved in cancer have recently been studied intensively. ${ }^{2-4}$ The use of gene expression data to predict carcinogenesis holds promise in GC diagnosis and prognosis. Thus, novel prognostic and diagnostic factors that are associated with $\mathrm{GC}$ progression would be of great clinical relevance.

The E2F transcription factors are key participants in a number of cellular events such as cell cycle, DNA synthesis or nuclear transcription. The E2F family of transcription factors is composed of activator (E2f1-3a) and repressor (E2f3b, 4-8) factors and is predominantly regulated by the $\mathrm{Rb}$ family of proteins (Rb, p107 and p130), 5,6 and the activating E2F transcription factors E2F1, E2F2 and E2F3 are central to regulation of the cell cycle genes. ${ }^{7}$ E2F1 is the most thoroughly investigated member of the E2F family in human malignancies. E2F1 has pivotal roles in tumor progression by modulation of both coding and non-coding transcripts, ${ }^{8,9}$ and was reported to act as oncogenes or tumor suppressors to modulate tumorigenesis depending on different cell context. $^{8,10,11}$ Accumulating evidence revealed E2F1 exert important effect on GC progression; however, the biology functions remain argued. ${ }^{12-14}$
TINCR, a long non-coding RNA (IncRNA) producing a 3.7-kb transcript, was first reported to bind to staufen1 (STAU1) protein and mediate differentiated mRNA stabilization. ${ }^{15}$ STAU1 protein is a double-stranded RNA-binding protein, and has various roles in gene expression. STAU1 binds to an STAU1-binding site in the $3^{\prime}$-untranslated region (3'-UTR) of its target mRNAs inducing mRNA degradation, which is termed STAU1-mediated mRNA decay (SMD). ${ }^{16}$ SMD is a translation-dependent mechanism that occurs when STAU1, together with the nonsense-mediated mRNA decay factor UPF1, is bound sufficiently downstream of a termination codon. ${ }^{16}$ Recently, we found that the expression of TINCR was elevated at the mRNA levels in GC cells and tissues and the upregulation of TINCR is induced by the transcription factor SP1. ${ }^{17}$ TINCR regulates cell growth, cell cycle progression by affecting $K L F 2$ mRNA stability via SMD. ${ }^{17}$

Here we report a novel pathway involved in E2F1 and TINCR in tumor development and GC cell growth. In this study, we found that: (a) E2F1 could promote GC proliferation and cell cycle progression; (b) patients with high E2F1 expression in their GC cells have a poor prognosis; (c) E2F1 could induce TINCR transcription activation; and (d) TINCR forces cell growth, cell cycle progression by affecting CDKN2B mRNA stability via SMD.

\footnotetext{
${ }^{1}$ Department of Oncology, The First Affiliated Hospital of Nanjing Medical University, Nanjing, P.R. China; ${ }^{2}$ Department of Pathology, The Affiliated Hospital of Yangzhou University, Yangzhou University, Yangzhou, P.R. China; ${ }^{3}$ State Key Laboratory of Microbial Metabolism, and School of Life Science and Biotechnology, Shanghai Jiaotong University, Shanghai, P.R. China; ${ }^{4}$ Department of Oncology, Jining No.1 People's Hospital, Jining City, China; ${ }^{5}$ Department of Medical Oncology, Huai'an First People's Hospital, Nanjing Medical University, Huai'an City, P.R. China; ${ }^{6}$ Department of Medical Laboratory, Nanjing Chest Hospital, Nanjing, P.R. China and ${ }^{7}$ Department of Biochemistry and Molecular Biology, Nanjing Medical University, Nanjing, P.R. China

*Corresponding author: Y-Q Shu, Department of Oncology, The First Affiliated Hospital, Nanjing Medical University, No. 300 Guangzhou Road, Nanjing, Jiangsu 210009, China. Tel: +86 25 68217221; Fax: +86 258372 4440; E-mail: yongqian_shu@163.com

${ }^{8}$ These authors contributed equally to this work and should be regarded as joint first authors.

Received 11.1.17; revised 25.2.17; accepted 28.2.17; Edited by E Candi
} 
a

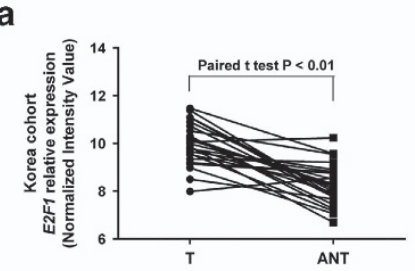

C
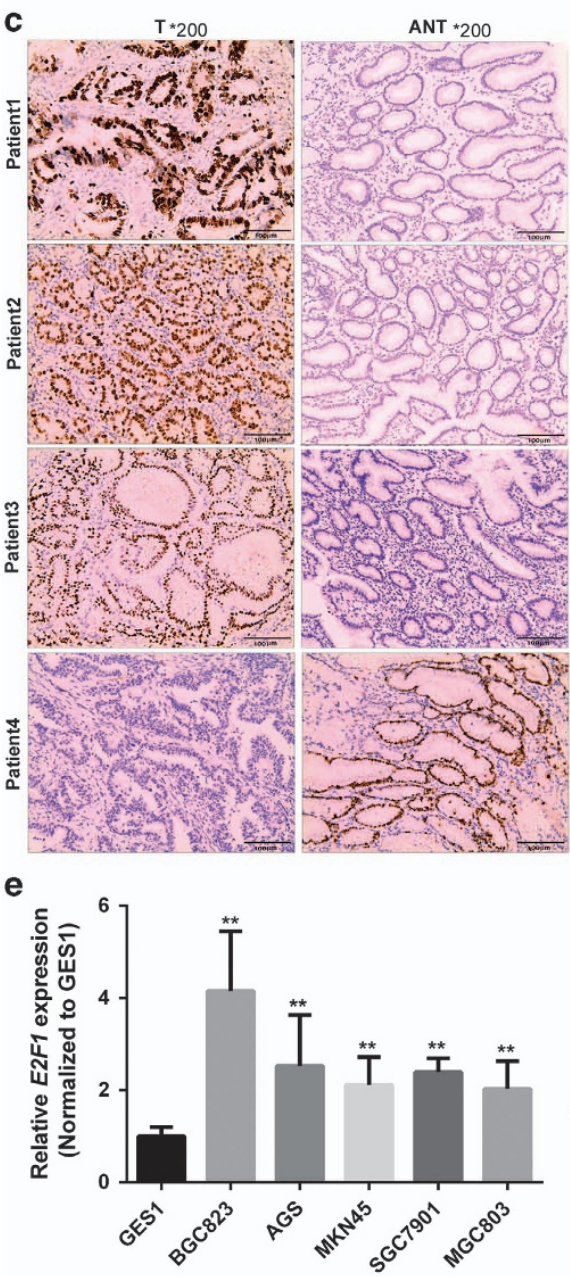

f

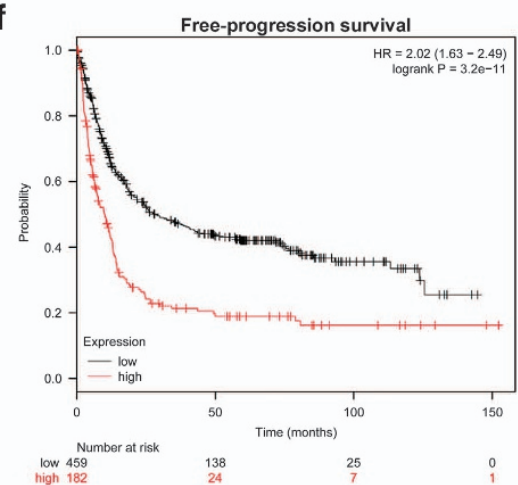

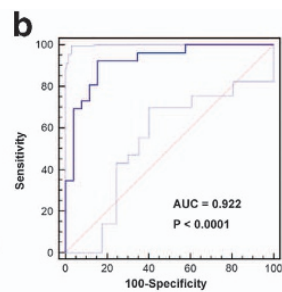

d
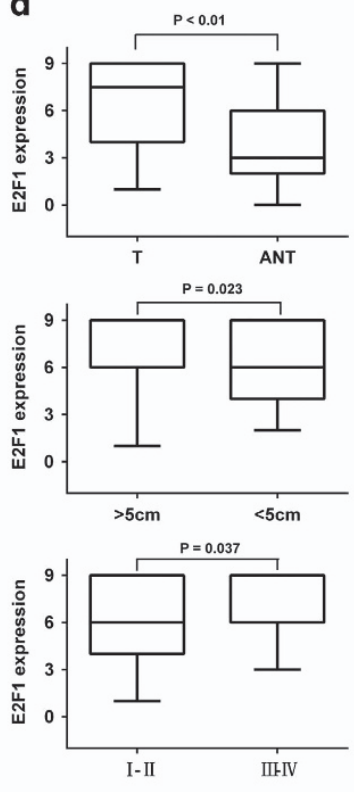

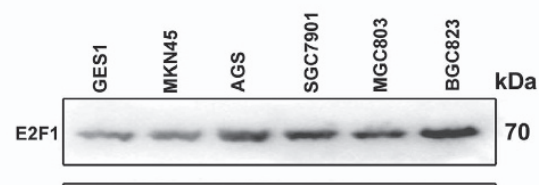
GAPDH $-\infty-\infty=0-17$

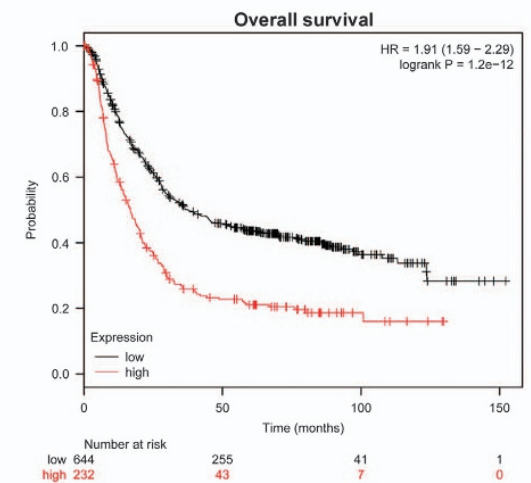




\section{Results}

E2F1 is overexpressed in GC tissues and cell lines, and upregulation of E2F1 indicate poor outcome of GC. To investigate the role of E2F1 in the progression of human $\mathrm{GC}$, a human microarray data sets (GSE51575) (26 paired cancer and noncancer tissues) was obtained to analyze E2F1 mRNA expressed between GC and paired non-tumor tissues. The result showed that E2F1 mRNA was 3.34-fold higher in gastric tumor tissues ( $\mathrm{T}$ ) compared with paired adjacent a

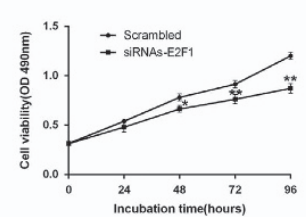

C
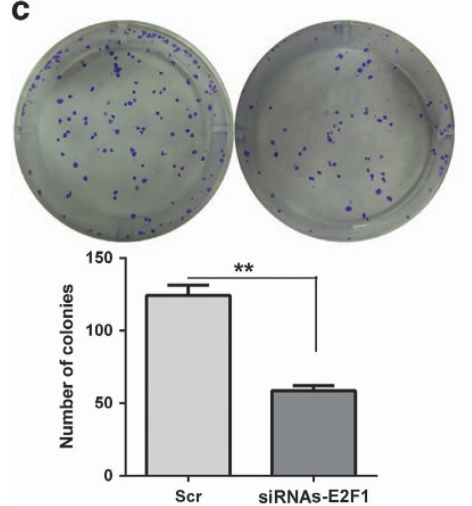

b

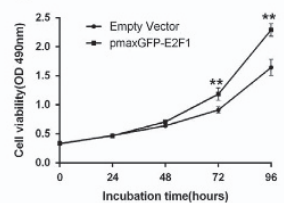

d
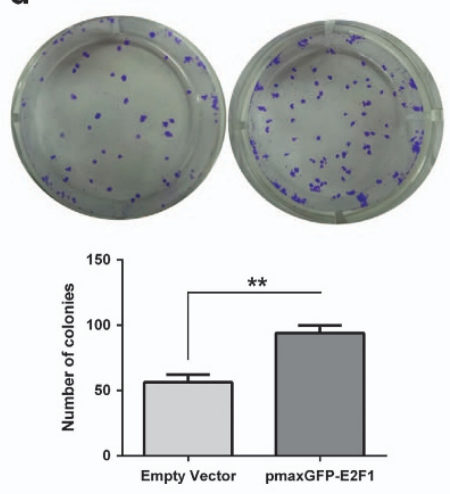

f
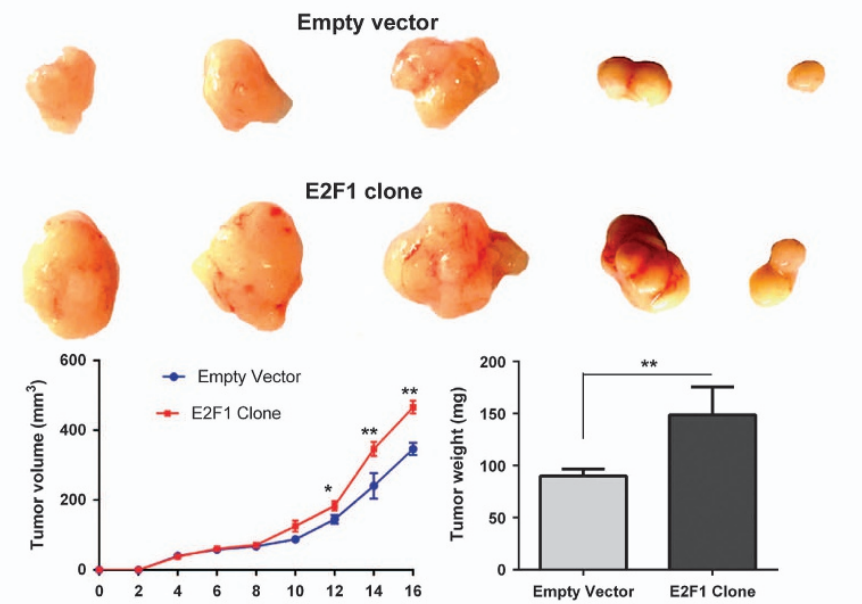

Figure 2 Functional roles of E2F1 in vitro and in vivo. E2F1 knockdown in GC cells transfected with siRNAs against E2F1 or E2F1 upregulation by pmaxGFP-E2F1 vector. E2F1 depletion inhibits GC cell growth, as detected by the (a) MTT assay and (c) colony-formation assay, whereas ectopic expression of E2F1 promotes GC cell growth, as examined by the (b) MTT assay and (d) colony-formation assay. Bars: S.D.; ${ }^{\star} P<0.05,{ }^{\star *} P<0.01$. (e) Cell cycle analyses in the BGC823 and MGC803 cell lines. Relative to scrambled siRNA-transfected cells, E2F1 knockdown induced significantly increased the number of cells in the G0/G1 phase and reduced the number of cells in the $S$ phase. Relative to empty vector-transfected cells, E2F1 upregulation promotes cell cycle progression. Representative FACS images and statistics based on three independent experiments. Bars: S.D.; ${ }^{*} P<0.05,{ }^{* *} P<0.01$. (f) Representative data showed that overexpression of E2F1 significantly promote tumor growth in nude mice xenograft model. MGC803 cells were transfected with empty vector or E2F1 expression vector and then injected into mouse flanks. Tumor growth was measured every 2 days after injection, and tumors were harvested at day 16 and weighed. (g) Detection of the cell proliferation markers PCNA in xenograft tumors by IHC

Figure 1 E2F1 is overexpressed in GC tissues and cell lines. (a) Analysis of E2F1 mRNA expression in GC and paired ANTs based on GSE51575 microarray database. (b) The ROC curve for prediction of GC based on E2F1 expression level in GSE51575, using corresponding adjacent non-tumorous tissues as a control. (c) IHC analysis of E2F1 protein expression in GC tumor tissues (T) and paired ANTs. Pictures of representative areas were presented at different staining intensities in ANT and T. (d) Analysis of the expression pattern of E2F1 in gastric tissues detected by IHC. Stages I-IV, TNM stages. Statistical analyses were performed using Student's paired $t$-test and one-way ANOVA. (e) Real-time RT-PCR and western blot analyzed the expression of E2F1 in a series of human GC cell lines and normal gastric epithelial cell line (GES-1). (f) Kaplan-Meier survival plots demonstrating the good prognostic effect of E2F1 upregulation correlated with a worse FPS and OS in GC patients $(n=876)$ 
normal tissues (ANTs) (Figure 1a). We plotted a receiver operating characteristic (ROC) curve with the non-tumorous tissues adjacent to the tumor tissues as a control based on GSE51575 database. The cutoff value for predicting GC tissues from normal tissues was 8.91 (normalized intensity value). The area under the ROC curve (AUC) was 0.922 (95\% confidence interval $(\mathrm{Cl})=0.813-0.978, P<0.0001)$, with the sensitivity and specificity were 0.923 and 0.846 , respectively (Figure 1b). We further confirmed E2F1 expression levels between clinical gastric tumors $(T)$ and paired ANTs from 80 cases of GC patients by immunohistochemistry (IHC) in our cohort. Our results showed that E2F1 was predominantly located in the nucleus of GC cells (Figure 1c). E2F1 expression found in GC tissues was significantly higher than in their adjacent tissues $(P<0.001$, Figure $1 \mathrm{~d}$ and Supplementary Table S2). We also confirmed that E2F1 expression was significantly increased in larger tumors $(P=0.023)$ and advanced TNM stages $(P=0.037$, Figure 1d). We further evaluated the expression levels of E2F1 in GC cell lines. The results showed that the expression levels of E2F1 were significantly increased in all tumourigenic GC cell lines than that in non-tumourigenic cell lines (Figure 1e). In addition, E2F1 expression is positively associated with FP (free progression) (hazard ratio $(\mathrm{HR})=$ 2.02; 95\% Cl, 1.63-2.49; $P<0.001)$ and overall survival (OS) $(\mathrm{HR}=1.91 ; 95 \% \mathrm{Cl}, 1.59-2.29 ; P<0.001)$ in $\mathrm{GC}$, which was supported by Kaplan-Meier plotter analysis (www.kmplot. com), using microarray data from 876 GC patients ${ }^{18}$ (Figure 1f).

Functional roles of E2F1 as a tumor activator in vitro and in vivo. To elucidate whether E2F1 could have a role in accelerating GC progression, gain- and loss-of-function approaches were used to evaluate the biological function of E2F1 in GC cell lines. We used chemically synthesized small interfering RNAs (siRNAs) to knockdown endogenous E2F1 in BGC823, which have relative high E2F1 expression. In addition, E2F1 was overexpressed by transfecting the pmaxGFP-E2F1 vector into MGC803 cell lines, which have relative low E2F1 expression. The depletion and ectopic expression of E2F1 in cells was confirmed by western blot (Supplementary Figure S1A). MTT ((3-(4, 5-dimethylthiazol-2yl)-2, 5-diphenyltetrazolium bromide) tetrazolium) and colony formation assays revealed that cells transfected with siRNAs but not scrambled in BGC823, had significantly inhibited growth and proliferation of GC cells (Figures $2 a$ and c). Meanwhile, ectopic overexpression of E2F1 by transfecting the MGC803 cell lines with the pmaxGFP-E2F1 vector, selected by the addition of G418, significantly promoted GC cell proliferation in vitro (Figures $2 \mathrm{~b}$ and $\mathrm{d}$ ). We also examined the effects of E2F1 on GC cell cycle progression. As illustrated in Figure 2e, inhibition of E2F1 markedly blocked the cell cycle at the G1-S phase, whereas overexpression of E2F1 promotes cell cycle progression. We extended the study of the E2F1 growth promotion role to in vivo athymic (nu/nu) mouse models, the results showed that E2F1-transfected cells developed significantly larger tumors than empty vectortransfected cells (Figure 2f). IHC staining analyses showed that alteration of E2F1 expression significantly changed the expression of the cell proliferation markers proliferating cell nuclear antigen (PCNA) in gastric cells (Figure $2 \mathrm{~g}$ ).

E2F1 upregulate TINCR expression in GC cells. Accumulating data revealed that E2F1 promote cancer progression by activation transcription of downstream oncogene in both coding and non-coding regions of the genome. Our previous study identified a IncRNA, TINCR, promotes GC proliferation and overexpression of TINCR indicates worse prognosis of GC. To unravel whether TINCR was regulated by E2F1 expression in GC, we examined the TINCR core promoter region for transcription factor binding sites, and identified six tandem putative E2F1-binding sites at the regions -366 to -355 bp (E1), -257 to -239 bp (E2), -136 to -124 bp (E3), -41 to -30 bp (E4), -16 to 0 (E5) and +56 to +73 bp (E6) in the TINCR promoter (Figure $3 \mathrm{a}$ ). We cloned the human TINCR promoter fragment (nucleotides -1000 to +163 ) into pGL3 vector for a luciferase activity assay. TINCR transcriptional activity was induced by E2F1 overexpression (Figure 3a). The results suggested that E2F1 participate in TINCR transcription regulation. To validate this finding, we deleted these binding sites individually and used them repeated as the reporter assay. The results showed that the deletion of the E2F1-binding motif E6 significantly impaired the effect of E2F1 on TINCR transcription activation, suggesting that E2F1 binds to their special binding motifs to regulate TINCR transcription (Figure $3 b$ ). To corroborate this notion, we performed in vivo chromatin immunoprecipitation (ChIP) assays to address whether E2F1 bind to the TINCR promoter region. The ChIP assay revealed that endogenous E2F1 bound to the TINCR promoter (Figure 3c). We next determined whether the overexpression of TINCR is mediated by E2F1, we applied loss- and gain-of-function approaches. We showed that the ectopic expression or siRNA knockdown, respectively, increased or reduced E2F1 enrichment on the TINCR promoter (Figure 3c), and resulted, respectively, in TINCR upregulation or downregulation in GC cells (Figure $3 d$ ). The correlation of E2F1 and TINCR gene transcription were further elucidated in tissues sample, and the result revealed that of TINCR expression is positively correlated with E2F1 mRNA levels in $\mathrm{GC}$ (Pearson $\mathrm{R}=0.469$, $P<0.001$ ) (Figure $3 e$ ). Hence, these results suggest that E2F1 serve as the transcriptional factors to activate TINCR transcription and upregulate its expression.

Overexpression of TINCR is potentially involved in the tumor promotion function of E2F1. Our previous work found that TINCR could promote GC cell line BGC823 and SGC7901 proliferation. ${ }^{17}$ Here, we further confirm the result in MGC803 and AGS cell lines. We used chemically synthesized siRNAs to knockdown endogenous TINCR in MGC803 and AGS cell lines, which both were considered appropriate for TINCR depletion (Supplementary Figure S1B). MTT assays show that siRNA transfectionmediated TINCR knockdown resulted in a significant decrease in cell viability rate in MGC803 and AGS, which tend to exhibit naturally high TINCR expression levels (Figure 4a). These observations were further confirmed by EDU (red)/DAPI (blue) immunostaining assay (Figure 4b). To investigate whether TINCR was involved in the E2F1-induced 
a
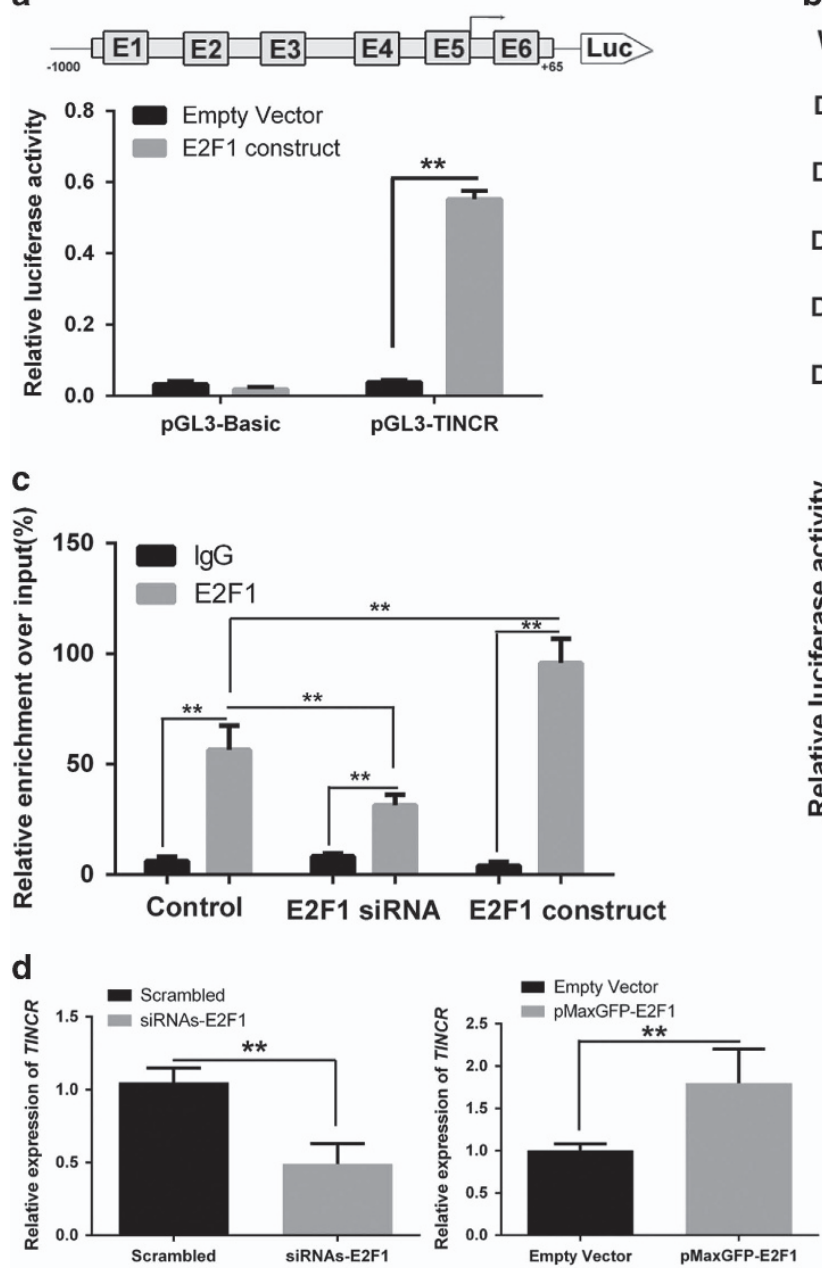

b
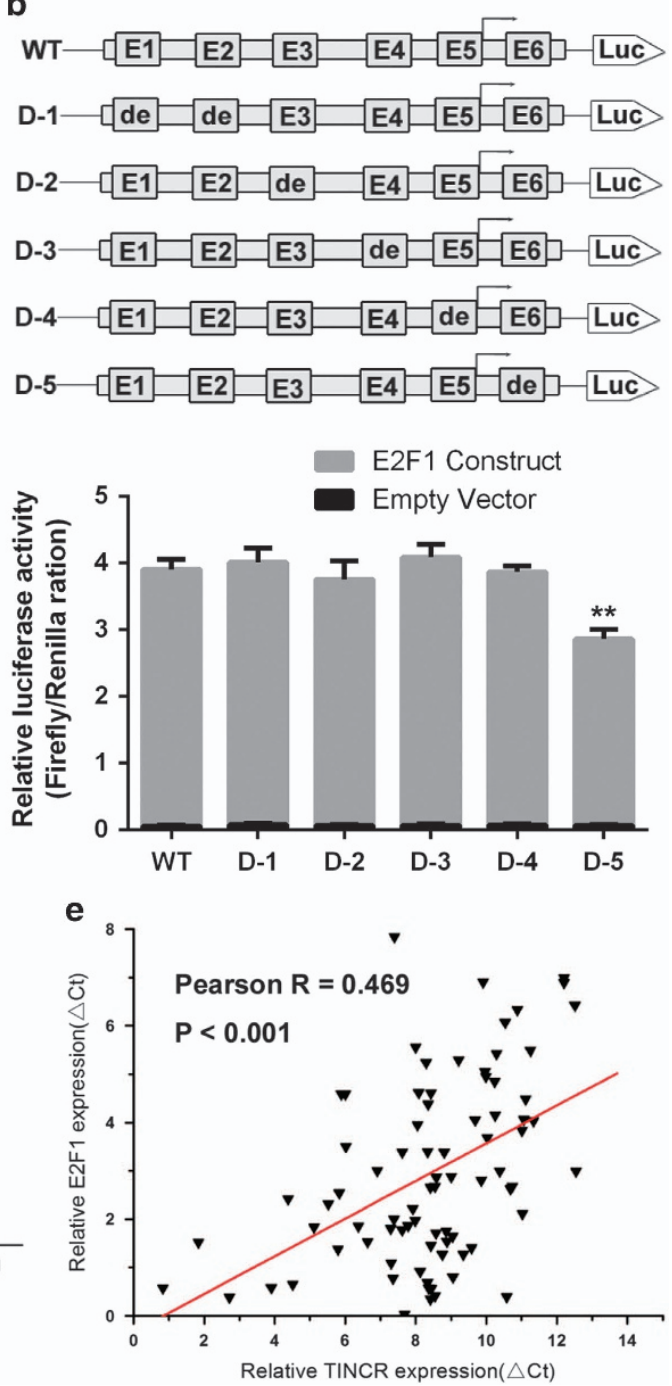

Figure 3 E2F1 upregulate TINCR expression in GC cells. (a) A dual-luciferase reporter assay was performed by co-transfection of the TINCR promoter fragment (TINCRpGL3) with overexpression of E2F1. (b) Reporter assay in cells transfected with various TINCR promoters constructs with deletion in different binding elements for E2F1 (WT, wild type; D, deletion type). Luciferase activity was expressed as relative to that of the pGL3 vector (a promoter-less vector) (c) ChIP assay demonstrated endogenous E2F1 binding to the TINCR gene promoter, and the ectopic expression or siRNA knockdown, respectively, increased or reduced E2F1 enrichment on the $T I N C R$ promoter ${ }^{*} P<0.05$, ${ }^{* *} P<0.01$. (d) qPCR analysis of TINCR expression levels following the treatment of BGC823 and MGC803 cells with siRNA-E2F1 and pmaxGFP-E2F1 expression vector, respectively. Bars: S.D.; ${ }^{*} P<0.05,{ }^{\star \star} P<0.01$. (e) Analysis of the relationship between TINCR expression ( $\Delta$ Ct value) and E2F1 mRNA level $(\Delta C$ t value) in $80 \mathrm{GC}$ tissues

increase in GC cell proliferation, we carried out rescue experiments. After transfection with si-TINCR, MGC803 cells were co-transfected with pmaxGFP-E2F1. MTT assays indicated that the co-transfection could partially rescue pmaxGFP-E2F1-promoted proliferation in MGC803 cells. (Figure 4c). Moreover, we found that co-transfection of pmaxGFP-E2F1 could rescue the upregulated expression of $C D K N 2 B$ protein induced by the depletion of TINCR (Figure 4d). These data indicated that E2F1 promotes GC cell proliferation partly through the upregulation of TINCR expression.

TINCR targets CDKN2B by SMD. Our previous study revealed that most TINCR molecules are located within the cytoplasm, and are bound to STAU1 protein in GC cells, and the results are further confirmed in MGC803 and AGS cell lines (Supplementary Figure S2). KLF2 mRNA was detected as a bona fide SMD target, which was mediated by TINCR in GC cells in our recent publication. We hypothesized that CDKN2B, which was elevated upon TINCR depletion, may also be direct TINCR-STAU1 complex targets. First, we analyzed the RNA interactome analysis data followed by deep sequencing (RIA-sequencing) provided by online GEO data sets (http://www.ncbi.nlm.nih.gov/geo/query/acc.cgi? acc $=$ GSE40121), and found that $C D K N 2 B$ are also bound to TINCR mRNA (Supplementary Table S3). And the binding regions are located at the $3^{\prime}-\mathrm{UTR}$ region of $C D K N 2 B$ mRNA (Supplementary Figure S3A). Previous evidence confirmed that TINCR interacts with mRNA through a 25-nucleotide motif that was strongly enriched in 
TINCR-interacting mRNAs and also repeated within TINCR itself, termed the TINCR box (Supplementary Figure S3B). ${ }^{19}$ We also speculated the CDKN2B sequence bound to the TINCR box (Supplementary Figure S3C).

a

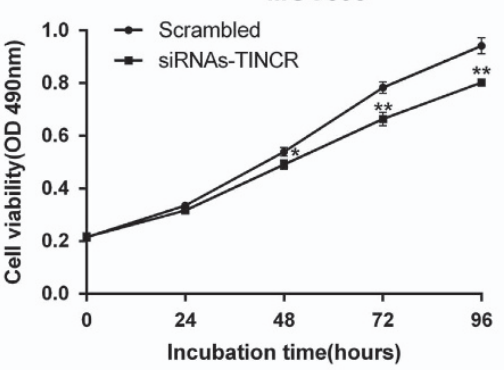

b

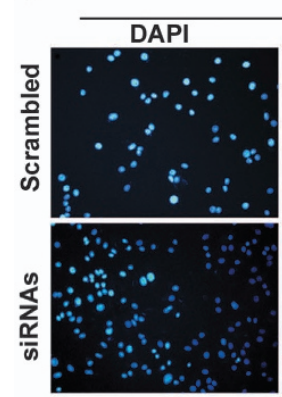

MGC803
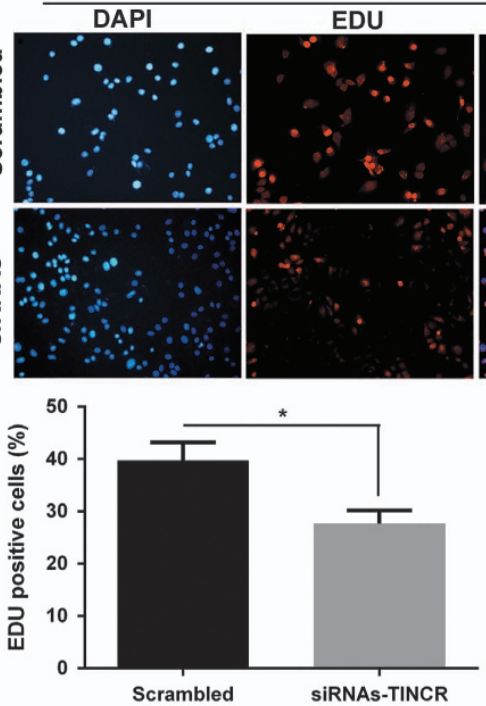

In order to confirm the above speculation, we performed in vitro assays in $\mathrm{GC}$ cells. First, we knockdown endogenous TINCR and STAU1 in GC cells, which both were considered appropriate depletion (Supplementary Figure S1B and C), and
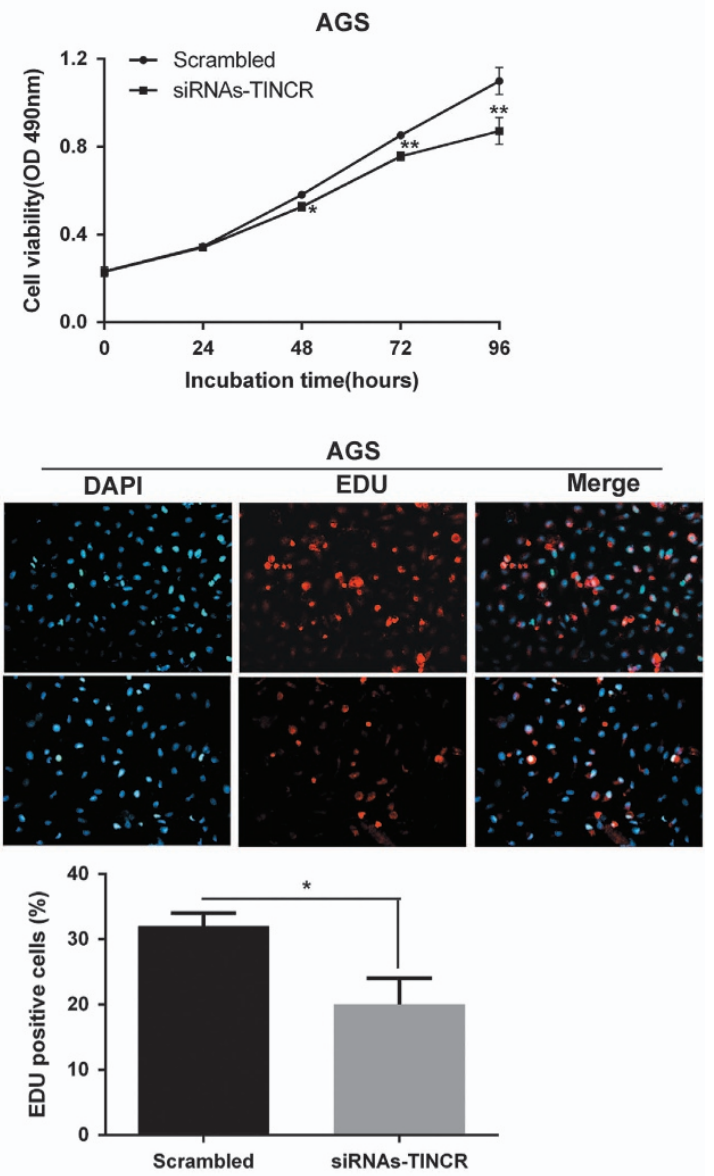

C

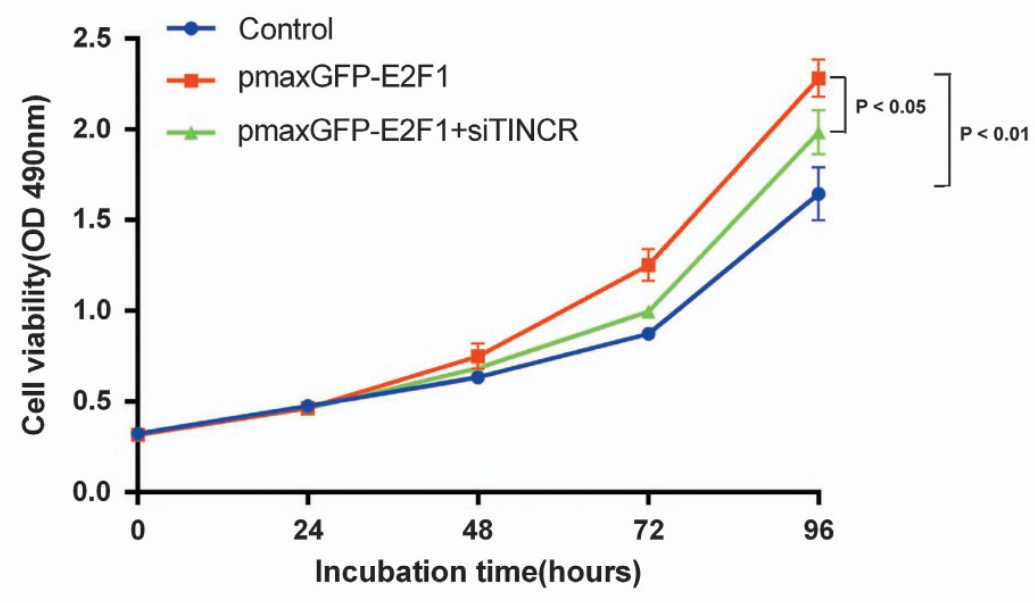

d Control si-TINCR pmaxGFP-E2F1 siTINCR+pmaxGFP-E2F1

kDa

CDKN2B

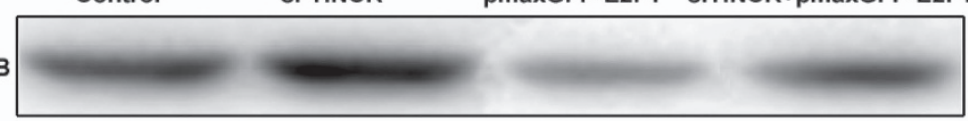

GAPDH

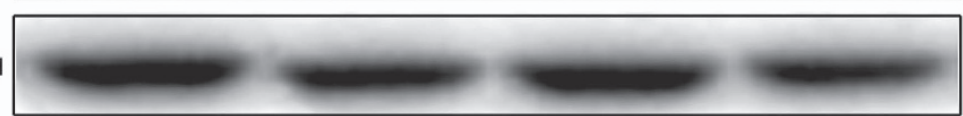


the abundance of $C D K N 2 B$ mRNA increased upon TINCR and STAU1-depleted GC cells (Figure 5a). Second, RNA immunoprecipitation (RIP) assays showed a remarkable enrichment of CDKN2B by STAU1 antibody compared with IgG control, indicating STAU1 could bind to CDKN2B mRNA (Figure $5 b$ ). Third, we determined whether the binding regions are located in $3^{\prime}$-UTRs, and cells were transfected with these test plasmids: pLUC-CDKN2B 3'-UTR, the STAU1-FLAG expression vector, pLUC-ARF1 SBS, and phCMV-MUP reference plasmid, which encodes major urinary protein (MUP) mRNA. The two latter of these served as a positive and a negative control, respectively, for STAU1-FLAG binding. ${ }^{16}$ Anti-FLAG could immunopurifiy Rluc-CDKN2B 3'-UTR, endogenous TINCR and Rluc-ARF1 SBS, but not MUP mRNA (Figure $5 \mathrm{c}$ ). Those results indicate that $C D K N 2 B$ is a bona fide SMD target in GC cells.

To further determine whether TINCR is required for the co-IP of STAU1 with CDKN2B mRNA, MGC803 cells that transiently transfected with control siRNA or siRNA against TINCR were immunoprecipitated using anti-STAU1 antibody. Compared with control siRNA, siRNA-TINCR reduced by $\sim 2$-fold the co-IP of STAU1 with CDKN2B mRNA (Figure 5d). Furthermore, the RNA pull-down assay revealed that TINCR interacted with CDKN2B mRNA (Figure 5e), and the depletion of STAU1 significantly reduced the interaction of TINCR with CDKN2B mRNA (Figure 5f), corroborating that STAU1 is required for the association between TINCR and CDKN2B mRNA. More importantly, the CDKN2B mRNA half-life was significantly increased upon downregulation of STAU1 or TINCR, whereas it was decreased after TINCR overexpression (Figure $5 \mathrm{~g}$ ). Our findings suggest that TINCR affects CDKN2B mRNA stability and expression through SMD.

\section{Discussion}

Recent findings have suggested that E2F family proteins have important roles in human malignancies. ${ }^{10} \mathrm{E} 2 \mathrm{~F} 1$, a key regulator for the G1/S phase transition in the E2F family, ${ }^{20}$ was reported to upregulate in GC. ${ }^{14}$ However, the function role in GC progression remains disagreed. In this study, we found that E2F1 expression was significantly upregulated in GC tissues compared with corresponding non-cancerous tissues. Specifically, E2F1 expression levels could be used to discriminate the cancer tissues from non-tumorous tissues. Moreover, patients with higher E2F1 levels appeared to have a greater tumor size, higher tumor stage and shorter survival than the lower group. Our results indicate that E2F1 expression provided a significantly predictive value and prognostic marker for patients with GC.

Our data revealed that silencing E2F1 expression led to significant inhibition of cell proliferation, whereas E2F1 overexpression contributed to cell growth and tumorigenicity. Knockdown of E2F1 expression contributed to G1 phase arrest and an $S$ phase reduction, whereas ectopic overexpression of E2F1 promoted cell cycle progression. Accumulation data revealed that E2F1 exert cell cycle modulation function by regulation of both coding and non-coding transcripts. A novel IncRNA, named TINCR, a potent cell cycle modulator in GC was identified in our recent work. Hence, we speculate that E2F1 and TINCR occurrence of mutual reaction. In this study, we found E2F1 could bind around +56 to $+73 \mathrm{bp}$ of TINCR promoter region and specifically activated its transcription. The G1-S transition in the cell cycle in mammalian cells is controlled by cyclins, cyclin-dependent kinases (CDKs), and their inhibitors, and deregulation of CDK inhibitors is a common feature in tumor cells. ${ }^{21}$ CDKN2B serve as potent growth inhibitors of cell cycle checkpoints. $^{21}$ Notably, consistent with our recent report, $C D K N 2 B$ was found to be remarkably upregulated upon TINCR or E2F1 knockdown in MGC803 and AGS cells. Taken together, CDKN2B could be crucial TINCR and E2F1 target.

LncRNAs can act together with specific proteins to perform various functions depending on their subcellular location, ${ }^{22,23}$ and TINCR is a predominantly cytoplasmic IncRNA in GC cells, indicating its action in post-transcriptional gene regulation. The results of RNA IP and RNA pull-down assays show that TINCR could bind STAU1, which is consistent with our previous data. ${ }^{17}$ STAU1 is a cytoplasmic protein and exerts multiple effects as a post-transcriptional regulator. Our teams have identified that TINCR targets KLF2 transcript through TINCR-STAU1 complex formation. Here, this study found that $C D K N 2 B$ is also a target of STAU1. In addition, $C D K N 2 B$ mRNA stability and the effects of binding to STAU1 are influenced by TINCR depletion. As evidenced above, TINCR may affect CDKN2B expression through SMD by TINCR-STAU1 complex formation. The pathway via which E2F1 and TINCR regulate cell cycle and cells proliferation has been depicted in Figure 6. The nuclear transcription factor E2F1 induces TINCR overexpression. TINCR recruits STAU1 to the $3^{\prime}$-UTR of CDKN2B mRNA, degrading CDKN2B through the UPF1-dependent mRNA decay mechanism. Subsequently, $C D K N 2 B$ depletion promotes cell cycle progression and tumorigenicity. Here, we explored a novel pathway involved in E2F1, TINCR and CDKN2B in GC development.

We describe here a novel mechanism underlying GC cell proliferation through a molecular cross talk between E2F1, TINCR, STAU1 and CDKN2B. Further insights into the functional and clinical implications of the pathway may contribute to early GC diagnosis and help with GC treatment.

\footnotetext{
Figure 4 TINCR promotes cells proliferation and regulates CDKN2B expression in MGC803 and AGS cells; and is involved in the E2F1-mediated promotion of viability. (a) MTT assays were performed to determine the cell viability of siRNAs-TINCR-transfected GC cells. (b) EDU (red)/DAPI (blue) immunostaining assay was used to confirm the results of MTT assay. The data represent the mean \pm S.D. from three independent experiments. ${ }^{*} P<0.05,{ }^{* *} P<0.01$. (c) The simultaneous depletion of $T I N C R$ could partly 'rescue' the proliferation effects induced by overexpressed E2F1 in MGC803 cells. Error bars represent S.D., $n=3 .{ }^{*} P<0.05$; ${ }^{* *} P<0.01$. (d) $T$ TINCR depletion upregulates the expression of CDKN2B in GC cells, and the simultaneous overexpression of E2F1 could partly 'rescue' the CDKN2B expression. The expression of CDKN2B protein in MGC803 cells was analyzed by western blotting
} 

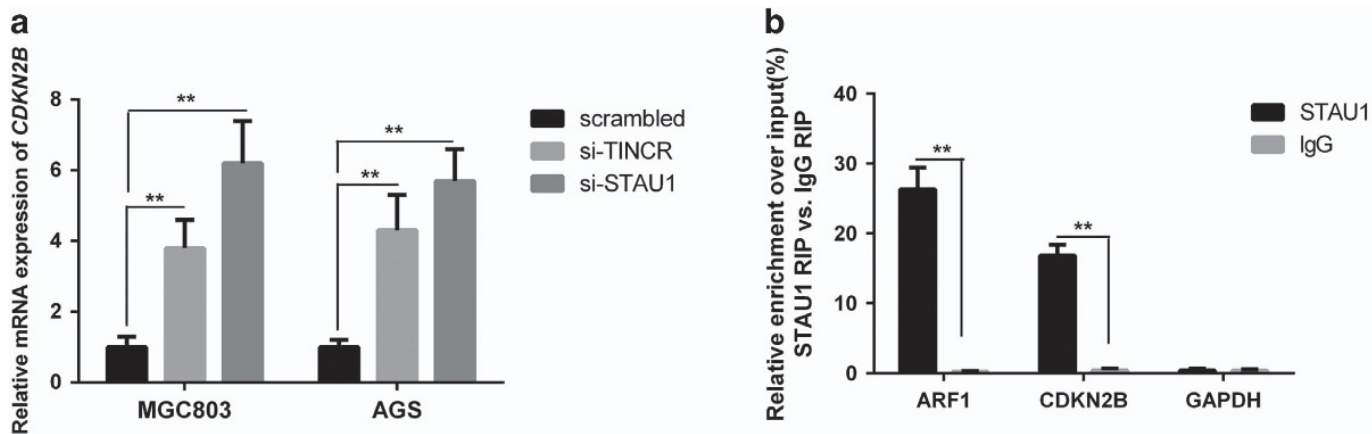

C
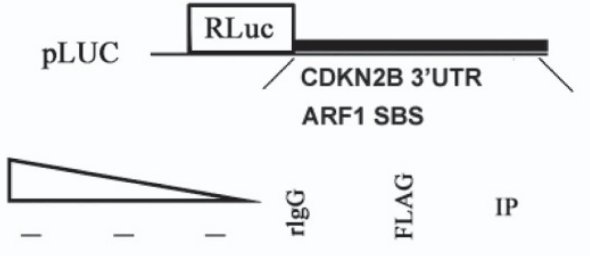

e
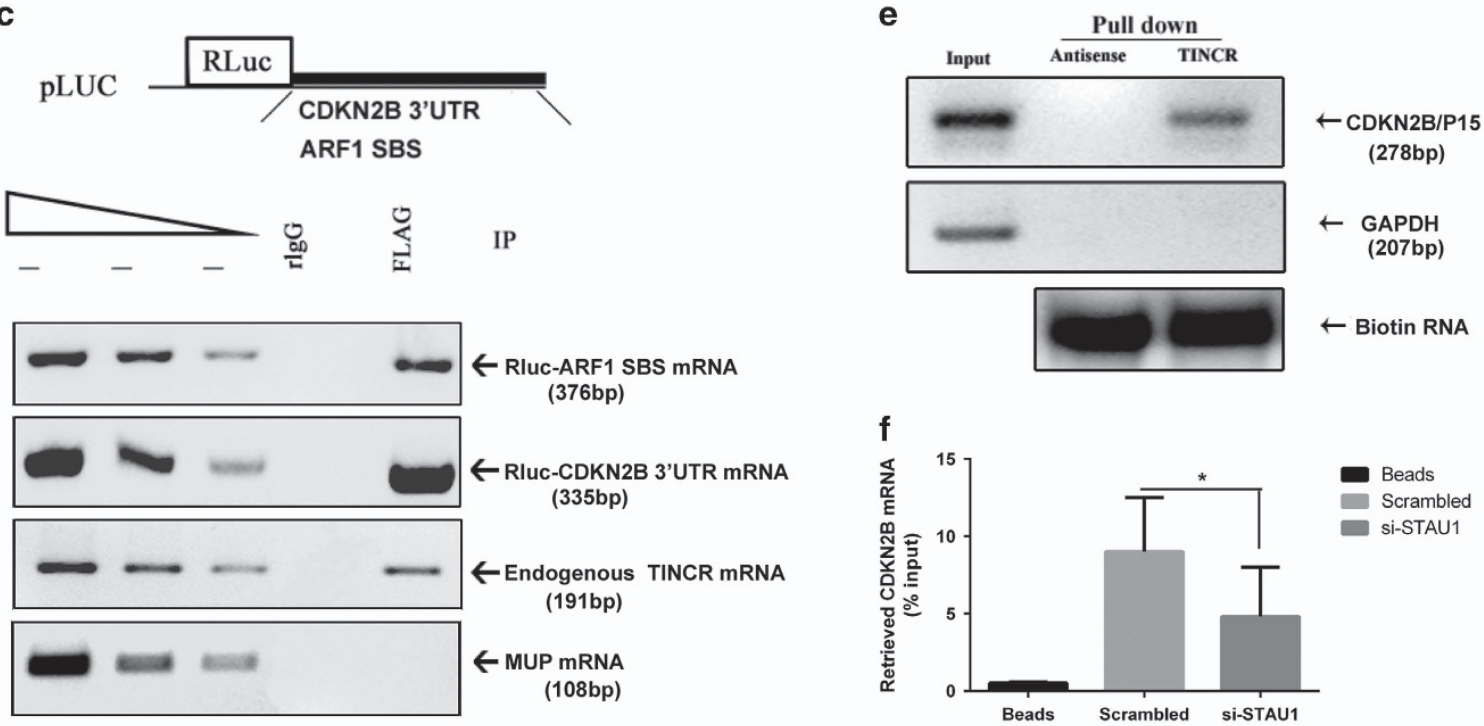

$\leftarrow$ Rluc-ARF1 SBS mRNA (376bp)
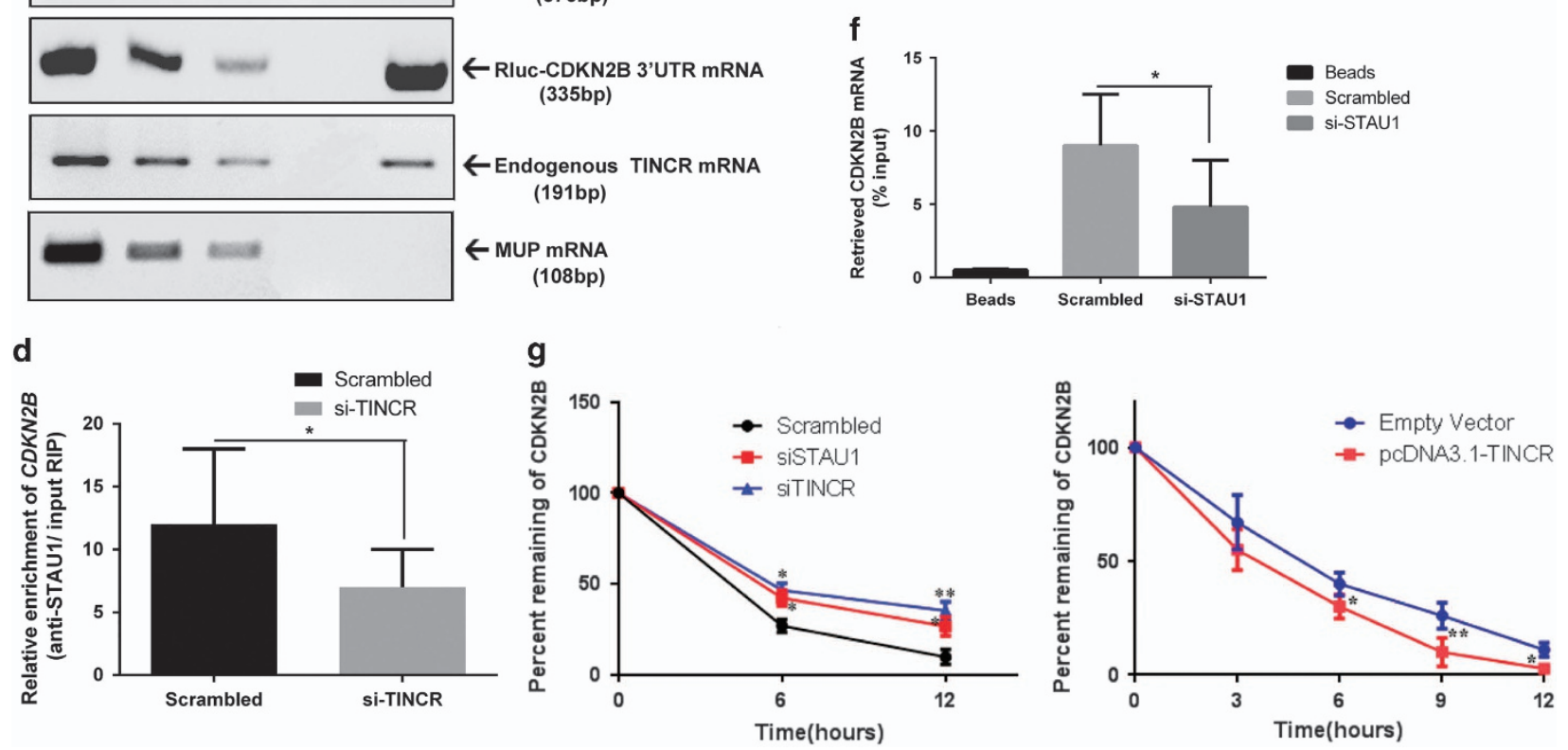

Figure 5 TINCR-STAU1 complex binds to CDKN2B mRNA and regulates their stability. (a) The abundance of CDKN2B mRNA was elevated upon TINCR and STAUI depletion in GC cells, detected by qRT-PCR. Error bars represent S.D., $n=3 .{ }^{* \star} P<0.01$. (b) Interaction of CDKN2B mRNA with STAU1, detected by RIP assay (the relative ARF1 enrichment served as a positive control, and the GAPDH as a negative control that did not interact with STAU1). Error bars represent S.D., $n=3$. ${ }^{* *} P<0.01$. (c) IP of STAU1FLAG. MGC803 cells were transiently co-transfected with (1) STAU1-FLAG expression plasmid; (2) RLuc-CDKN2B 3'-UTR; (3) phCMV-MUP, which encodes MUP mRNA that lacks an SBS and serves as a negative control for STAU1-FLAG binding; and (4) Rluc-ARF1 SBS, which contains an ARF1 SBS downstream of the translation termination codon of C-terminally deleted renilla luciferase and serves as a positive control for STAU1-FLAG binding. After cell lysis, total RNA and protein were purified from the lysate before and after IP using FLAG antibody or nonspecific rabbit ( $r$ ) IgG. The three leftmost lanes represent two-fold serial dilutions of RNA and demonstrate that the RT-PCR is semiquantitative. Schematic representations of the pLUC-CDKN2B 3'-UTR and pLUC-ARF1 SBS test plasmids (above). RT-PCR analysis demonstrates that CDKN2B 3'-UTRs, endogenous TINCR, and ARF1 SBS bind STAU1-FLAG, whereas MUP mRNA does not (below). Results are representative of three independently performed experiments. (d) Inhibiting CDKN2B mRNA interacting with STAU1 upon TINCR depletion, detected by RIP experiments. MGC803 cells were transfected with control (Scrambled) or si-TINCR, and cellular extract was prepared for RIP assay using SATU1 antibody $24 \mathrm{~h}$ after transfection. Error bars represent S.D, $n=3$. ${ }^{*} P<0.05$. (e) Biotinylated TINCR RNA pulls down the full-length CDKN2B mRNA detected by RT-PCR analysis. A nonspecific RNA (GAPDH) is shown as a control. (f) STAU1 depletion reduced the interaction between TINCR with CDKN2B mRNA. MGC803 cells were transfected with control (Scrambled) or si-STAU1, and cell lysates were incubated with biotin-labeled TINCR; after pull-down, mRNAs were extracted and assessed by qRT-PCR. Error bars represent S.D., $n=3 .{ }^{*} P<0.05 ;{ }^{* *} P<0.01$. (g) TINCR or STAU1 control CDKN2B mRNA stability. RNA stability assays were performed in MGC803 cells using Actinomycin D to disrupt RNA synthesis degradation rates of the mRNA CDKN2B over $12 \mathrm{~h}$. ${ }^{*} P<0.05 ;{ }^{*} P<0.01$ 


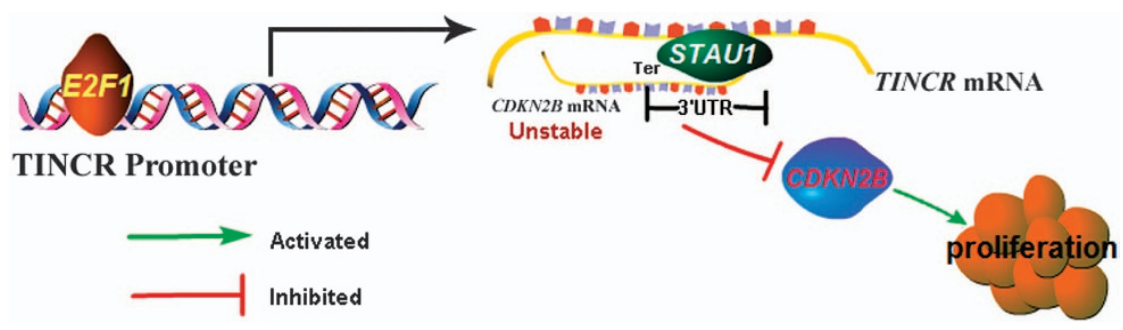

Figure 6 Summary diagram describes that E2F1 and TINCR regulates GC cell proliferation

\section{Materials and Methods}

Plasmids, RNA interference and transfection. pmaxGFP-E2F1 expression vector was purchased from Addgene (plasmid \#16007) (Cambridge, USA). To construct Rluc-CDKN2B 3'-UTR and Rluc-ARF1 SBS, pLuc luciferase vector (Ambion Inc., Grand Island, NY, USA) carrying the Renilla luciferase (Rluc) reporter gene was digested with an endonuclease and ligated to the corresponding fragment that encodes human CDKN2B $3^{\prime}$-UTR and ARF1 SBS mRNA. The CDKN2B $3^{\prime}$-UTR and ARF1 SBS mRNA fragments were amplified by PCR using a CDNA library from MGC803 cells as a template and the primers listed below: for CDKN2B $3^{\prime}$-UTR: $5^{\prime}$ cacaactcgagCACCCCCACCCACCTAATTC-3' (sense) and $5^{\prime}$-tgaagatctTGCC AGGTGGCTTCGAAAAT- 3 ' (antisense), where the bold nucleotides specify the $X$ Xol site, and the italic underlined nucleotides specify the Bgll site.

For ARF1 SBS mRNA: 5'-cacaagtcgacGTGAACGCGACCCCCCTCCCTCTC ACTC-3' (sense) and 5'-aaggatcCCCAGGTGCCCATGGGCCTACATCCCC-3' (antisense), where the bold nucleotides specify the Sall site, and the italic nucleotides specify the BamHI site. To construct the luciferase reporter vectors, the core promoter of the TINCR gene $(-1000$ to +163 , relative to the transcription start site of the TINCR gene) and the relative deletion of binding sites were respectively subcloned into the pGL3 basic firefly luciferase reporter. siRNAs for specifically knockdown E2F1, TINCR and STAU1 were chemically synthesized (Invitrogen, Shanghai, China), and the sequences of the oligonucleotides synthesized for RNAi have been listed in Supplementary Table S1. Transfections were carried out using Lipofectamine 2000 reagent according to the manufacturer's instructions (Invitrogen, Shanghai, China).

Cell lines and immunoblot analysis. The human gastric adenocarcinoma cancer cell lines MGC803, BGC823, MKN45, AGS and SGC7901 and the normal gastric epithelium cell line (GES-1) were obtained from the Chinese Academy of Sciences Committee on Type Culture Collection Cell Bank (Shanghai, China). Western blot analysis was conducted according to our previous protocol. ${ }^{24}$ Antibodies used in the study were: E2F1 (cat. \# ab14768, Abcam, Hong Kong, China), CDKN2B (cat. \# Sc-271791, Santa Cruz, Dallas, TX, USA), STAU1 (03-116, Millipore, Bedford, MA, USA), FLAG-tagged antibodies (8146 S, Cell Signaling Technology, Boston, MA, USA), and GAPDH antibody was used as control.

Tissue samples and clinical data collection. In this study, 80 patients underwent primary GC resection at the First Affiliated Hospital of Nanjing Medical University and the Affiliated Hospital of Yangzhou University. The study was approved by the ethics committee on Human Research of the First Affiliated Hospital of Nanjing Medical University and the Affiliated Hospital of Yangzhou University. Written informed consent was obtained from all patients. The clinicopathological characteristics of the GC patients have been summarized in Supplementary Table S2.

RNA preparation and quantitative real-time PCR. Total RNAs were extracted with TRIzol reagent (Invitrogen, Grand Island, NY, USA), and quantitative real-time PCR (qRT-PCR) analyses were conducted according to the manufacturer's instructions (Takara, Dalian, China). The primers sequences have been listed in Supplementary Table S1.

Isolation of cytoplasmic, and nuclear RNA. Cytoplasmic and nuclear RNA were isolated and purified using the Cytoplasmic \& Nuclear RNA Purification Kit (Norgen, Belmont, CA, USA), according to the manufacturer's instructions.
IHC analysis. To quantify protein expression, both the intensity and extent of immunoreactivity were evaluated and scored. In the present study, staining intensity was scored as follows: 0 , negative staining; 1 , weak staining; 2 , moderate staining; and 3 , strong staining. The scores of the extent of immunoreactivity ranged from 0 to 3 , and were determined according to the percentage of cells that showed positive staining in each microscopic field of view $(0,<25 \% ; 1,25-50 \% ; 2,50-75 \% ; 3$, $75-100 \%)$. A final score ranging from 0 to 9 was achieved by multiplying the scores for intensity and extent. Using this method, the expression of proteins was scored as $0,1,2,3,4,6$ or 9 . In case of disagreement (score discrepancy 0.1 ), slides were reexamined and a consensus was reached by the experts.

Luciferase reporter assay. Cells were first transfected with appropriate plasmids in 24-well plates. Next, the cells were collected and lysed for luciferase assay $48 \mathrm{~h}$ after transfection. The relative luciferase activity was normalized with Renilla luciferase activity.

Cell proliferation assays. Cell proliferation assays and colony formation assays were performed as previously reported. ${ }^{24}$

Flow cytometry. Cell cycle and cell apoptosis were analyzed by flow cytometry and detected as previously reported. ${ }^{24}$

EDU analysis. 5-Ethynyl-2-deoxyuridine (EDU) labeling/detection kit (Ribobio, Guangzhou, China) was used to assess the cell proliferation. Cells were grown in 96-well plates at $5 \times 103$ cells per well. Forty-eight hours after transfection, $50 \mu \mathrm{m}$ EdU labeling media were added to the 96-well plates and they were incubated for $2 \mathrm{~h}$ at $37^{\circ} \mathrm{C}$ under $5 \% \mathrm{CO}_{2}$. After treatment with $4 \%$ paraformaldehyde and $0.5 \%$ Triton X-100, cells were stained with anti-EdU working solution. DAPI was used to label cell nuclei. The percentage of EdU-positive cells was calculated after analyses of fluorescent microscopy. Five fields of view were randomly assessed for each treatment group.

Chromatin immunoprecipitation. ChIP assays were performed using the EZ ChIPTM Chromatin Immunoprecipitation Kit (Millipore), according to the manual. The primer sequences were listed in Supplementary Table S1.

RIP and RNA pull-down. We performed RIP experiments using the Magna RIP RNA-Binding Protein Immunoprecipitation Kit (Millipore) according to the manufacturer's instructions. The STAU1 and FLAG-tagged antibodies used for IP were from Millipore (03-116; RIPAb+ STAU1) and Cell Signaling Technology (8146S), respectively. The details of the primers for RT-PCR and qPCR have been provided in Supplementary Table S1.

Biotin-labeled RNAs were transcribed in vitro with the Biotin RNA Labeling Mix (Roche Diagnostics, Shanghai, China) and T7 RNA polymerase (Roche Diagnostics), treated with RNase-free DNase I (Roche Diagnostics) and purified with an RNeasy Mini Kit (Qiagen, Valencia, CA, USA). Next, 1 mg whole-cell lysates from MGC803 cells was incubated with $3 \mu \mathrm{g}$ of purified biotinylated transcripts for $1 \mathrm{~h}$ at $25^{\circ} \mathrm{C}$. Complexes were isolated with streptavidin agarose beads (Invitrogen, Grand Island, NY, USA). The beads were washed briefly three times and boiled in sodium dodecyl sulfate buffer, and the retrieved protein was detected using the standard western blot technique. The RNA present in the pull-down material was detected using reverse transcription polymerase chain reaction (RT-PCR) and qPCR analysis. The RT-PCR and qPCR primer pairs were provided in Supplementary Table S1. 
RNA stability assay. To analyze RNA stability, GC cells were treated with actinomycin $D(1 \mu \mathrm{g} / \mathrm{ml})$. Cells were collected at different time points, and RNA was extracted using Trizol reagent (Invitrogen, Grand Island, NY, USA). Reverse transcription was performed using oligo (dT) primers and mRNA levels were measured using qRT-PCR.

Bioinformatics analysis and statistical analysis. GC gene expression data was obtained from the NCBI GEO, (http://www.ncbi.nlm.nih.gov/geo/). One data set GSE51575 consisted of 26 paired primary gastric adenocarcinoma tissues and surrounding normal fresh frozen tissues was included. All the tissues were obtained after curative resection and pathologic confirmation at Samsung Medical Center (Korea cohort). The raw CEL files from the Agilent arrays (Agilent, Santa Clara, CA, USA) for GSE51575 were processed and normalized using the Robust Multichip Average as previously described. ${ }^{25}$ All statistical analyses were performed using SPSS 20.0 software (IBM, SPSS, Chicago, IL, USA). The significance of differences between groups was estimated using the Student's t-test, $\chi 2$ test, Fisher's exact test, Mann-Whitney test, Kruskal-Wallis test or Wilcoxon test, as appropriate. A ROC curve was established to evaluate the diagnostic value for differentiating between $\mathrm{GC}$ and benign diseases. FP survival (FPS) and OS rates were calculated by the KaplanMeier method with the log-rank test applied for comparison. Pearson correlation analysis was performed to investigate the correlation between TINCR and E2F1 mRNA expression. Two-sided $P$-values were calculated, and a probability level of 0.05 was chosen for statistical significance.

\section{Conflict of Interest}

The authors declare no conflict of interest.

Acknowledgements. This study was supported by the National Natura Science Foundation of China (81672896 and 81602071), Jiangsu Province Clinical Science and Technology projects (Clinical Research Center, BL2012008), Natura Science Foundation of Jiangsu Province for Youth (BK20161066) and the Priority Academic Program Development of Jiangsu Higher Education Institutions (Public Health and Preventive Medicine, JX10231801).

1. Torre LA, Bray F, Siegel RL, Ferlay J, Lortet-Tieulent J, Jemal A. Global cancer statistics, 2012. CA Cancer J Clin 2015; 65: 87-108.

2. Jenkins BJ, Grail D, Nheu T, Najdovska M, Wang B, Waring P et al. Hyperactivation of Stat3 in gp130 mutant mice promotes gastric hyperproliferation and desensitizes TGF-beta signaling. Nat Med 2005; 11: 845-852.

3. Cheng AS, Li MS, Kang W, Cheng VY, Chou JL, Lau SS et al. Helicobacter pylori causes epigenetic dysregulation of FOXD3 to promote gastric carcinogenesis. Gastroenterology 2013; 144: $122-133$ e129.

4. Palanisamy N, Ateeq B, Kalyana-Sundaram S, Pflueger D, Ramnarayanan K, Shankar S et al. Rearrangements of the RAF kinase pathway in prostate cancer, gastric cancer and melanoma. Nat Med 2010; 16: 793-798.

5. Chen HZ, Tsai SY, Leone G. Emerging roles of E2Fs in cancer: an exit from cell cycle control. Nat Rev Cancer 2009; 9: 785-797.

6. Burkhart DL, Sage J. Cellular mechanisms of tumour suppression by the retinoblastoma gene. Nat Rev Cancer 2008; 8: 671-682.

7. Bertoli C, Skotheim JM, de Bruin RA. Control of cell cycle transcription during G1 and S phases. Nat Rev Mol Cell Biol 2013; 14: 518-528.

8. Tarangelo A, Lo N, Teng R, Kim E, Le L, Watson D et al. Recruitment of Pontin/Reptin by E2f1 amplifies E2f transcriptional response during cancer progression. Nat Commun 2015; 6: 10028 .
9. Sun M, Nie F, Wang Y, Zhang Z, Hou J, He D et al. LncRNA HOXA11-AS promotes proliferation and invasion of gastric cancer by scaffolding the chromatin modification factors PRC2, LSD1 and DNMT1. Cancer Res 2016; 76: 6299-6310.

10. Engelmann D, Putzer BM. The dark side of E2F1: in transit beyond apoptosis. Cancer Res 2012; 72: 571-575.

11. Yamasaki L, Bronson R, Williams BO, Dyson NJ, Harlow E, Jacks T. Loss of E2F-1 reduces tumorigenesis and extends the lifespan of $\mathrm{Rb} 1(+/ /)$ mice. Nat Genet 1998; 18: 360-364.

12. Xie Y, Wang C, Li L, Ma Y, Yin Y, Xiao Q. Overexpression of E2F-1 inhibits progression of gastric cancer in vitro. Cell Biol Int 2009; 33: 640-649.

13. Xie $Y$, Yin $Y$, Li L, Ma Y, Xiao Q. Short interfering RNA directed against the E2F-1 gene suppressing gastric cancer progression in vitro. Oncol Rep 2009; 21: $1345-1353$.

14. Zhang X, Ni Z, Duan Z, Xin Z, Wang $\mathrm{H}$, Tan $\mathrm{J}$ et al. Overexpression of E2F mRNAs associated with gastric cancer progression identified by the transcription factor and miRNA co-regulatory network analysis. PLOS ONE 2015; 10: e0116979.

15. Kretz M, Siprashvili Z, Chu C, Webster DE, Zehnder A, Qu K et al. Control of somatic tissue differentiation by the long non-coding RNA TINCR. Nature 2013; 493: 231-235.

16. Kim YK, Furic L, Desgroseillers L, Maquat LE. Mammalian Staufen1 recruits Upf1 to specific mRNA 3'UTRs so as to elicit mRNA decay. Cell 2005; 120: 195-208.

17. Xu TP, Liu XX, Xia R, Yin L, Kong R, Chen WM et al. SP1-induced upregulation of the long noncoding RNA TINCR regulates cell proliferation and apoptosis by affecting KLF2 mRNA stability in gastric cancer. Oncogene 2015; 34: 5648-5661.

18. Szasz AM, Lanczky A, Nagy A, Forster S, Hark K, Green JE et al. Cross-validation of survival associated biomarkers in gastric cancer using transcriptomic data of 1,065 patients. Oncotarget 2016; 7: 49322-49333.

19. Kretz M, Siprashvili Z, Chu C, Webster DE, Zehnder A, Qu K et al. Control of somatic tissue differentiation by the long non-coding RNA TINCR. Nature 2013; 493: 231-U245.

20. Hallstrom TC, Nevins JR. Balancing the decision of cell proliferation and cell fate. Cell Cycle 2009; 8: 532-535.

21. Sherr CJ, Roberts JM. Inhibitors of mammalian G1 cyclin-dependent kinases. Genes Dev 1995; 9: 1149-1163.

22. Tsai MC, Manor O, Wan $\mathrm{Y}$, Mosammaparast $\mathrm{N}$, Wang JK, Lan $\mathrm{F}$ et al. Long noncoding RNA as modular scaffold of histone modification complexes. Science 2010; 329: 689-693.

23. Martianov I, Ramadass A, Barros AS, Chow N, Akoulitchev A. Repression of the human dihydrofolate reductase gene by a non-coding interfering transcript. Nature 2007; 445 : $666-670$.

24. Xu TP, Huang MD, Xia R, Liu XX, Sun M, Yin L et al. Decreased expression of the long non-coding RNA FENDRR is associated with poor prognosis in gastric cancer and FENDRR regulates gastric cancer cell metastasis by affecting fibronectin1 expression. $J$ Hematol Oncol 2014; $7: 63$.

25. Irizarry RA, Hobbs B, Collin F, Beazer-Barclay YD, Antonellis KJ, Scherf U et al. Exploration, normalization, and summaries of high density oligonucleotide array probe level data. Biostatistics 2003; 4: 249-264.

cc) (i) Cell Death and Disease is an open-access journal published by Nature Publishing Group. This work is licensed under a Creative Commons Attribution 4.0 International License. The images or other third party material in this article are included in the article's Creative Commons license, unless indicated otherwise in the credit line; if the material is not included under the Creative Commons license, users will need to obtain permission from the license holder to reproduce the material. To view a copy of this license, visit http://creativecommons.org/licenses/by/4.0/

(C) The Author(s) 2017 